\title{
Research on MEMS Cantilever Beam Used as Switch in Thermal Mass Flow Meter
}

\author{
Pengyu Wang \\ Department of Electronic Information Engineering, Zhonghuan Information College, Tianjin \\ University of Technology, Tianjin, 300380, China
}

Keywords: Cantilever; MEMS; Flow meter; Switch

\begin{abstract}
In order to reduce power consumption of thermal mass flow meter and prolong its working life as long as possible, we suppose that a MEMS cantilever beam could be used as switch in thermal mass flow meter. First, we discussed working principle of cantilever beam to confirm the feasibility as switch in circuit. Second, we analyzed its property by using the simulation model. The simulation results show that the bending degree of cantilever beam is not only associated with young's modulus of materials, and also related to cantilever size such as length, thickness and length to width ratio. Therefore, if we can choice suitable material and design reasonable dimension, the MEMS cantilever beam may be used as switch.
\end{abstract}

\section{Introduction}

MEMS (Micro Electro Mechanical Systems) that refers to microelectronic and micromachining technology, integrates with micro sensors, micro actuators, signal processing, control circuit, communication interface circuit and the power supply, in smaller physical size, is a complete micro electromechanical system [1]. In the principle of thermal flow measurement, MEMS thermal mass flow meter is a sensor. It has many features such as fast response speed, large measurement range and high sensitivity.[2,3,4] Especially, for small flow detection sensitivity and good stability, it is suitable for industrial and civil application [5,6,7]. Most of the device supply is DC battery that has a problem of the energy shortage. Thus, it is necessary to consider energy-saving in the circuit design [8]. The power supply should be closed when there is no gas through and opened when the gas is through again. Then, the consumption of electrical energy reaches the minimum value.

Based on the above, the design of circuit switch using cantilever is feasible. We can use the static characteristics of the cantilever beam to open the circuit after the induction air flow generated. The cantilever makes the circuit to be closed when there is no air flow. Thereby it can reduce power consumption and the complexity of circuit design greatly.

\section{Working Principle}

The cantilever beam structure is shown in Fig.1. It is in a non-pressure state for position 1. For position 2, it is in external pressure state while the cantilever beam is resulted deformation and connected with the lower end of the electrode.

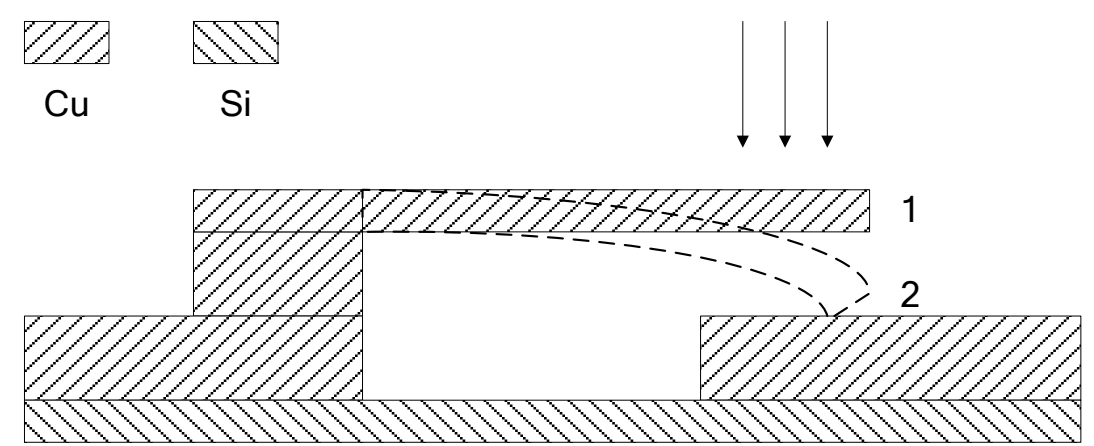

Fig.1: Mold of cantilever changed with force 
When there is a uniform distribution of stress $q$ in the cantilever beam, we can obtain the deflection as $\omega$ [9].

$$
\omega(x)=\frac{q x^{2}}{24 E I}\left(x^{2}-4 L x+6 L^{2}\right)
$$

If $\mathrm{x}=\mathrm{L}$, we can get the maximum deflection of the cantilever beam end:

$$
\omega(L)=\frac{q x^{2}}{8 E I}
$$

Where $\mathrm{x}$ is the distance from the measured point to the fixed end of cantilever, $\mathrm{L}$ is the length of cantilever, E is Young's modulus of material, I is inertia moment.

The structure of flow meter in aisle is shown in Fig.2. The switch is composed with a metal cantilever beam and a metal contact point. Put the cantilever in aisle, so that the bending direction is parallel to the direction of air flow. When air flow run through the aisle, the pressure makes the cantilever to be curved downward, the end of the cantilever contacts the metal point. Then the signal can be conducted, and the switch is in the "on" state. When there is no external air flow, the cantilever is restored to the initial state, so that the cantilever end may be divorced from the point, and the switch is in the "off" state. From Fig.2, we find that the switch could be equivalent to a series of micro strip gap of cantilever beam structure. We can establish equivalent circuit when the switch is in the "on" or "off" state.

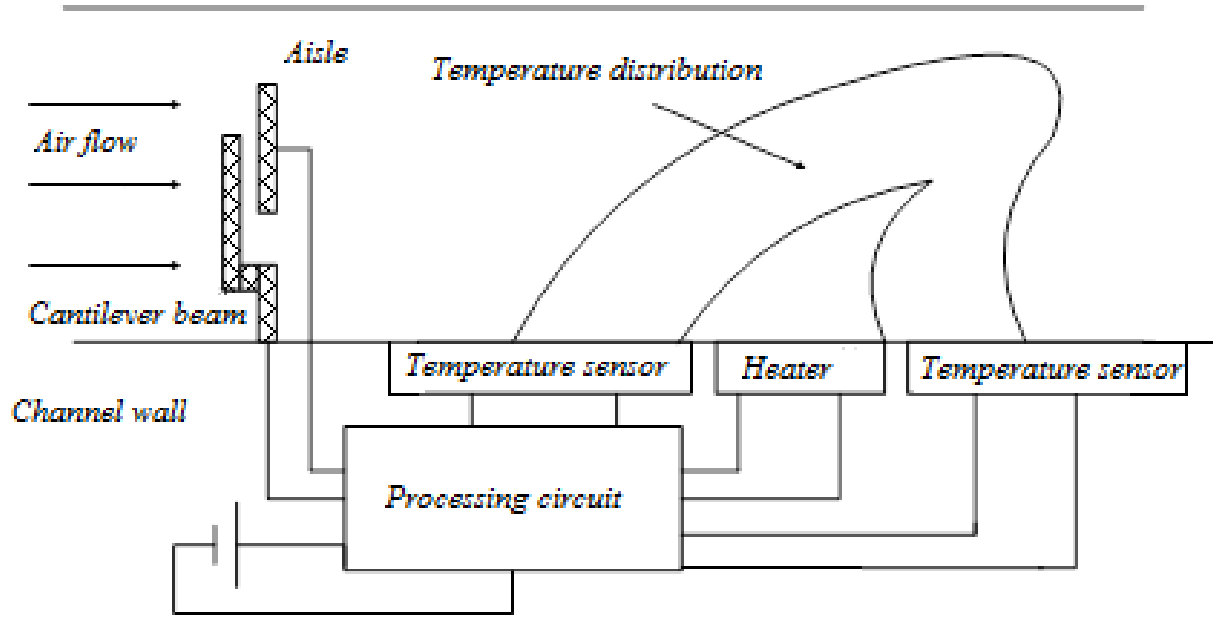

Fig.2: Structure of flow meter in aisle

\section{Device simulation}

We use the software intelliSuite to simulate cantilever beams model and make simulation. First, impose conditions that the air flow is added from the root to the tip of the beam on the device. Then the air flow causes deformation of the cantilever beam, in the microcosmic situation. The beam is curved downward and connected with the lower end nodes. Thus the circuit is conducted and the beam completes the role of switch [10,11,12 ]. Because the cantilever beam is perpendicular to the aisle, the effect of gravity could be ignored.

In the conditions pressing on the up surface of different material beam with the same pressure, we can see that the bending degree of the beam is different. The material parameters are shown in Table 1.

Simulation conditions:

cantilever beam thickness: $0.1 \mu \mathrm{m}$, length: $20 * 10 \mu \mathrm{m}$;

simulation pressure: $2 \mathrm{e}^{-3} \mathrm{Mpa}$. 
Table1: Setting and results of simulation

\begin{tabular}{lllll}
\hline \multicolumn{1}{c}{$\begin{array}{c}\text { Young's } \\
\text { modulus } \\
(\mathrm{GPa})\end{array}$} & $\begin{array}{l}\text { Poisson's } \\
\text { ratio }\end{array}$ & $\begin{array}{l}\text { density }\left(\mathrm{g} / \mathrm{cm}^{3}\right) \\
\mathrm{C}\end{array}$ & $\begin{array}{l}\text { the } \\
\text { shape } \\
\text { variable } \\
(\mu \mathrm{m})\end{array}$ \\
\hline $\mathrm{Cu}$ & 122.5 & 0.34 & 8.9 & 3.58 \\
$\mathrm{Al}$ & 68.85 & 0.36 & 2.7 & 6.29 \\
$\mathrm{Au}$ & 74.48 & 0.42 & 19.28 & 5.50 \\
$\mathrm{Pt}$ & 146.9 & 0.35 & 21.45 & 2.96 \\
\hline
\end{tabular}

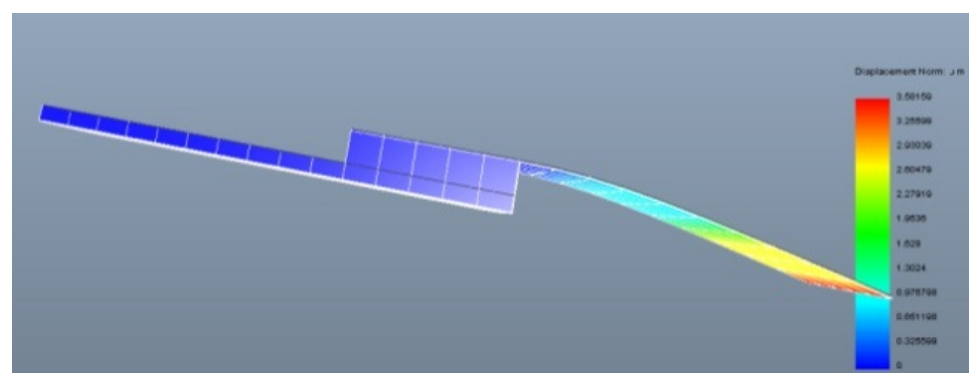

Fig.3 (a): $\mathrm{Cu}$

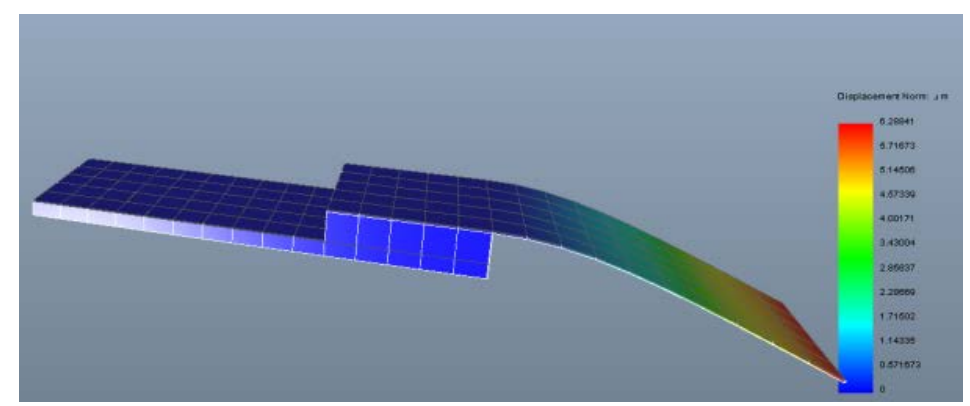

Fig.3 (b): Al

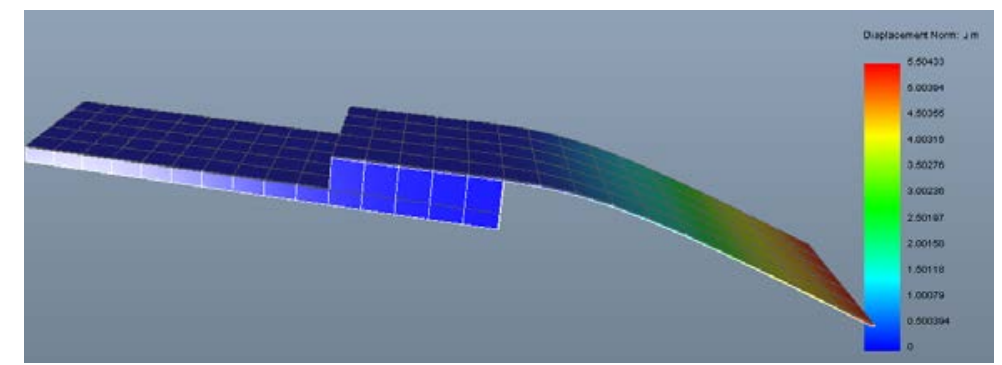

Fig.3(c): Au

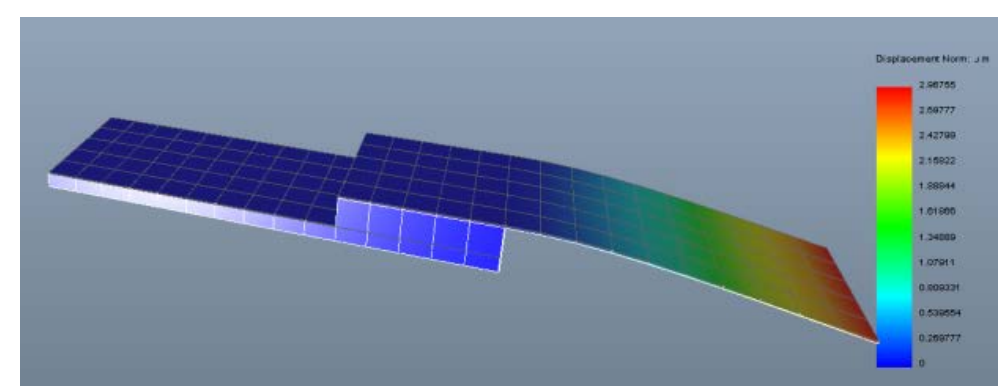

Fig.3(d): Pt

Fig.3: Results of simulation for different materials

Simulation results are shown in Fig.3. We can see that the bending degree of each material is different in the same condition. The copper bending degree is $3.58 \mu \mathrm{m}$. The aluminum bending 
degree is $6.29 \mu \mathrm{m}$. The gold bending degree is $5.5 \mu \mathrm{m}$. The platinum bending degree is $2.97 \mu \mathrm{m}$. Analysis data shows that the bending degree is closely related to young's modulus of material. In order to verify the results, we simulated bending degree of copper cantilever beam, in the case of other conditions were not changed except young's modulus. The results are shown in Table 2.

Table2: Simulation result of different young's modulus

\begin{tabular}{cccccc}
\hline $\begin{array}{c}\text { Young's } \\
(\mathrm{Gpa})\end{array}$ & modulus & 122.5 & 100 & 80 & 68.85 \\
\hline $\begin{array}{c}\text { Bending } \\
(\mu \mathrm{m})\end{array}$ & displacement & 3.58 & 4.39 & 5.48 & 6.29 \\
\hline
\end{tabular}

We find that bending degree of device is falling as young's modulus is increasing. When young's modulus is reduced to the extent of the aluminum material, the simulation result is the same as aluminum bending degree. We obtain a conclusion that material bending degree for the same structure is just associated with young's modulus of the material. At the same time we find:

$$
E * \omega=N
$$

Where $\mathrm{E}$ is young's modulus, $\omega$ is bending degree, $\mathrm{N}$ is constant.

Thus, the bending degree is inversely proportional to young's modulus of materials. Then we can verify the conclusion of Equation 2.

Fig. 4 shows the effect of cantilever beam size on the bending degree. In Fig.4 (a) the abscissa is the thickness and the ordinate is bending degree of the beam. The bending degree is falling when the film thickness is increasing. In order to meet the suitable bending degree, we should reduce the thickness of the beam as far as possible under the conditions allow. Then, the cantilever beam plays the role of switch with low pressure. In Fig.4 (b) the abscissa is the length and the ordinate is bending degree of the beam. The degree of bending is increasing exponentially as the increase of the cantilever beam length. Thus, the size has a decisive influence on the property of the cantilever beam.

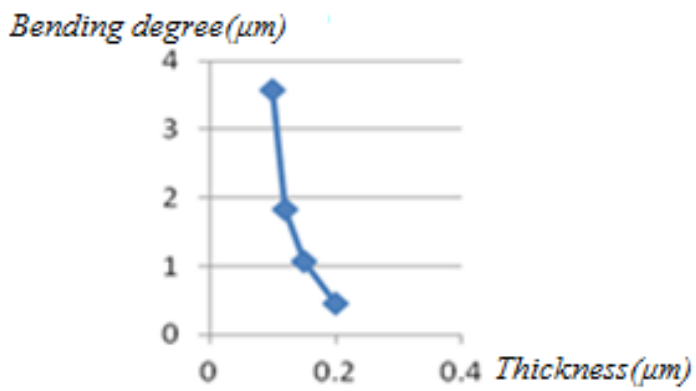

Fig.4(a): Thickness

Bending degree $(\mu \mathrm{m})$

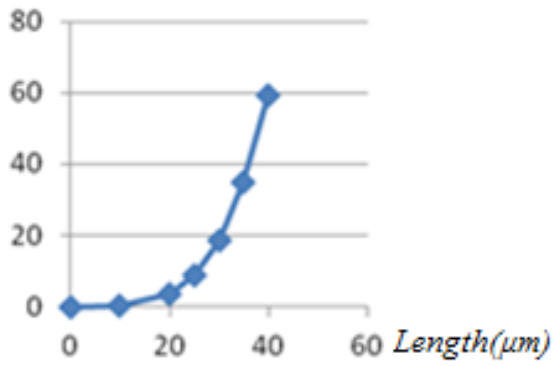

Fig.4 (b): Length

Fig.4 Effect of cantilever beam size on the bending degree

Examples of cantilever has different length to width ratio are shown in Fig.5. 
We find that the length to width ratio of fig.5(a) is larger than fig.5(b), but the bending degree of fig.5(a) is smaller than fig.5(b).
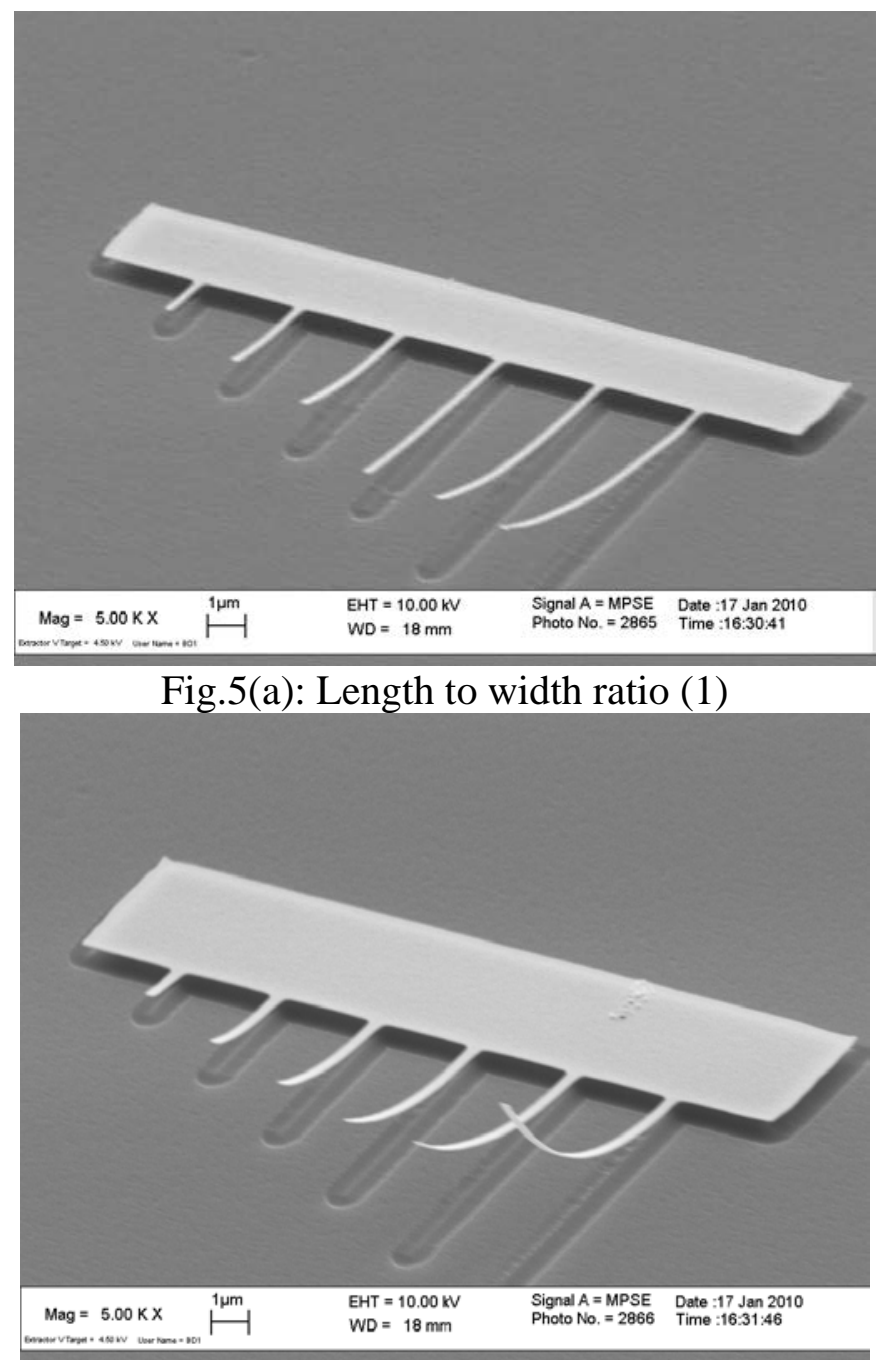

Fig.5(b): Length to width ratio (2)

Fig.5: Cantilevers with different length to width ratio

\section{Conclusions}

Based on the feasibility analysis of MEMS cantilever beam as switch, we researched on the property of the cantilever by simulation. The simulation results show that the bending degree of the cantilever decreases with the increase of Young's modulus. In addition, the bending degree decreases exponentially with the increases of the film thickness of the cantilever, and increases exponentially with the elongation of the cantilever. But, if the cantilever is too long, it will have some disadvantages such as anti-fatigue ability weakly. Thus, the cantilever size should be determined according to the actual situation. Therefore, the cantilever which is placed in the flow channel can be used as switch. Because the idle time of the flow channel is long, the power consumption can be reduced by this way.

\section{References}

[1] Liu Xiao-Bin, Analysis and research of MEMS progress. Mechanical Research \& Application, (15), pp.72-75, 2005. 
[2] Huang Shuo, Fu Xin, A numerical investigation of conjugate forced heat transfer for a micro thermal flow sensor,2009 IEEE/ASME International Conference on Advanced Intelligent Mechatronics Suntec Convention and Exhibition Center ,Singapore, pp.1510-1514,2009.

[3] Terao Minako, Akutsu Tomomi, Yoshiaki Tanaka, Non-wetted thermal micro flow sensor, SICE Annual Conference 2007, Japan: Kagawa University, pp.2084-2088, 2007.

[4] Honschoten Joost W van, Optimization of a thermal flow sensor for acoustic particle velocity measurements. Journal of Micro Electro Mechanical Systems, 14(3), pp. 436-443, 2005.

[5] Si Dong-hao, Li Yan-song, Thermal mass flow sensor based on technology of microelectronic mechanical systems (MEMS). Foreign Electronic Measurement technology, (1), pp.49-52, 2005.

[6] Bruschi Paolo, Dei Michele, Low-Power 2-D Wind Sensor Based on Integrated Flow Meters. IEEE Sensor Journal, pp. 1688-1696, December 2009.

[7] Wang Yu-hsiang, Chen Chang-pen. MEMS-based gas flow sensors. Microfluid Nanofluid, (6),pp.333-346,2009.

[8] Liang Guo-wei, Wen Ying-jie, Huang Zhen-wei, LIANG Yi-ling. Analysis and studies for measuring principle and affecting factors of hot type gas flowmeters. Journal of China Jiliang University, (19),pp.201-205,2008.

[9]Wang Zhe-yao. MEMS design and nanufacturing, Beijing: Tsinghua University Press, 2008.

[10] Schöler L, Lange B. Monolithically integrated micro flow sensor for lab-on-chip applications, Microelectronic Engineering,(79),pp.164-170, 2005.

[11] Buchner R, Maiwald M. Miniaturised thermal flow sensors for rough environments. Tech. Digest MEMS 2006, p.582-585,2006.

[12] Lee Chi-yuan, Lee Shuo-jen, Integration of micro temperature sensor and metal foil as gas diffusion layer for micro fuel cell, Proceedings of the 2009 4th IEEE International Conference on Nano/Micro Engineered and Molecular Systems, Shenzhen, China, pp.168-171,2009. 